\title{
Probing van der Waals and magnetic forces in bacteria with magnetic nanoparticles
}

\author{
Ana Lucía Campaña \\ Department of Physics \\ University of Oslo \\ Oslo, Norway \\ a.1.c.perilla@fys.uio.no \\ Anja Røyne \\ Department of Physics \\ University of Oslo \\ Oslo, Norway \\ anja.royne@fys.uio.no
}

\author{
Nadeem Joudeh \\ Department of Biosciences \\ University of Oslo \\ Oslo, Norway \\ nadeem.joudeh@ibv.uio.no \\ Dirk Linke \\ Department of Biosciences \\ University of Oslo \\ Oslo, Norway \\ dirk.linke@ibv.uio.no
}

\author{
Henrik Høyer \\ GIAMAG Technologies AS \\ Oslo, Norway \\ henrik@giamag.com \\ Pavlo Mikheenko \\ Department of Physics \\ University of Oslo \\ Oslo, Norway \\ pavlo.mikheenko@fys.uio.no
}

\begin{abstract}
Bioinspired metal-based nanoparticles have potential uses in many applications, but before a possible commercial exploitation, it is important to clarify the pathways of their production and deposition inside the organisms, for example in bacteria. The technique of magnetic force microscopy (MFM) could be used to evaluate the nanoparticles magnetic properties, in addition to allowing tracing their location inside or outside of bacteria, which could help to understand pathways of their biosynthesis. In this work, using MFM and analyzing the interaction of magnetic tip with nanoparticles and bacteria imbedded in resin at different heights above the surface, and comparing gradients of forces recorded by magnetic and non-magnetic tips, a condition was found, which allows to measure pure magnetic response of PdFe nanoparticles. For these nanoparticles, the interplay between magnetic and van der Waals forces is described at small distances to the surface. Experimental data are compared with simulations, based on the calculation of the distribution of magnetic field around a nanoparticle, which defines magnetic force acting on the MFM tip.
\end{abstract}

Keywords-Nanoparticles, magnetic force, van der Waals interaction, bacteria, magnetic force microscopy

\section{INTRODUCTION}

Magnetic nanoparticles have emerged as an attractive tool in many bio- and nanotechnology applications due to their unique properties defined by the state of their surface. Most magnetic nanoparticles are based on the period-4 transition metal elements $\mathrm{Fe}, \mathrm{Co}$ and $\mathrm{Ni}$, which show good magnetic properties at room temperature. Instead, palladium $(\mathrm{Pd})$, a period 5 element, which is positioned just below $\mathrm{Ni}$ in the periodic table of elements, is used in this work. Palladium is a paramagnetic metal on the verge of ferromagnetism in the bulk state that becomes ferromagnetic in nanoparticle form [1,2]. The big advantage of $\mathrm{Pd}$ in contrast to other magnetic elements is its high stability towards oxidation. Accepted by many as a noble metal, it resists entering chemical reactions itself and behaves as an excellent catalyst accelerating other chemical reactions [3]. The prospect of the simultaneous use of the magnetic and the catalytic properties of Pd nanoparticles (Pd NPs) would give extra benefit for the continuous development of magnetic nanoparticles applications, just as in cancer treatment, where studies have shown big potential of Pd NPs [4]

One biological route to produce magnetic nanoparticles consists of exposing bacteria to a solution containing positively charged ions of selected metals [5,6]. The nucleation of the nanoparticles takes place on the surface of bacteria, at points where they produce sufficient reduction potential in the form of electrons. The growth of nanoparticles could be accelerated by adding extra sources of reducing agents, for example, bubbling hydrogen through the solution or adding sodium formate to the medium. After being produced, the nanoparticle properties need to be tested. The magnetic properties can be evaluated, for example, by magnetometry [7], producing dry powder containing bacteria and nanoparticles and setting it in a magnetometer. However, this methodology does not allow to see the location of individual nanoparticles with respect to the bacteria, hampering the understanding of the metabolic routes used for their production. An alternative way is using Magnetic Force Microscopy (MFM), which, however, requires special preparation of samples with smooth surfaces down to the nanometer scale.

In this paper, being able to produce smooth surfaces, we employed the MFM technique for Pd NPs magnetic characterization. In the technique, a magnetic tip scans above the surface registering its interaction with the magnetic nanoparticles. In order to resolve the nanoparticles, the tip needs to be very close to the surface, at distances of a few nanometers. An obvious complication at these working distances is that the additional, van der Waals, force starts acting on the tip. Here, obtaining desired information from usual magnetic surface scanning methods is not a trivial task, as van der Waals force hinders the isolation of the magnetic force required in this study. To simplify the experiment, a small amount of Fe was added to the Pd NPs, which is known to increase the magnetism of Pd [8]. This adjustment allowed easy detection of the nanoparticle magnetism, by setting the tip at the distances at which the interfering van der Waals force was below the detection limit. 


\section{EXPERIMENTAL}

\section{A. Sample preparation}

A biological route was used for Pd-Fe nanoparticles (Pd$\mathrm{Fe}$ NPs) production. Nanoparticles were fabricated by preparing a dense suspension of BW25113 strain of the bacteria Escherichia coli and adding it to a solution of sodium tetrachloropalladate and equal molar amount of iron (III) chloride. After incubation for one hour at room temperature, sodium formate was added to accelerate nanoparticles growth. The tube with the solution was left until the suspension reached a dark black tone as indication for the complete reaction. Due to a very large difference in redox potential of $\mathrm{Pd}$ and $\mathrm{Fe}$, produced nanoparticles contained mainly $\mathrm{Pd}$ and a small amount of Fe. The resulting $\mathrm{Pd}-\mathrm{Fe}$ loaded bacteria were washed twice, centrifuged, fixed in a special solution, dehydrated and exposed overnight to a 1:1 mixture of acetone and epon. On the next day, the mixture was centrifuged again, and epoxy was added followed by incubation for one hour at room temperature. After that, the resulting mixture was incubated in an oven at $60{ }^{\circ} \mathrm{C}$ for 3 days.

The hardened sample was finally sliced with a diamond knife into 0.5 to $1 \mu \mathrm{m}$ thin slices. The slices were moved in a water droplet, put on smooth glass slides and left to rest for a few minutes for water evaporation. This resulted in the slices firmly attached to the slides, which were now ready to be measured by MFM.

\section{B. Magnetic force microscopy}

Magnetic force microscopy is a scanning probe microscopy technique, which uses a magnetized tip for mapping the magnetic properties of flat surfaces [9-11]. The technique is sensitive to transverse gradients of the vertical component of magnetic field and is suitable for measuring forces imposed by individual nanoparticles. In order to map the magnetism of a nanoparticle with high resolution, the tip should be placed very close to the surface, at a distance of few nanometers. At these distances, however, van der Waals force becomes strong, and it must be separated from the magnetic force.

A two-pass scanning technique $[9,10]$ presents a good solution to resolve this issue. In the first pass, the tip, which is operated in tapping mode at different frequencies specific for the employed tip, measures the surface topography. For this work a frequency close to $74 \mathrm{KHz}$ was used. In the following scans, the same topography is repeated at larger heights. Because the magnetic force decays slower away from the surface than the van der Waals force, a pure or dominant magnetic response of a nanoparticle can be registered above some distance away from the surface.

In tapping mode, the shift in the phase of oscillations of the cantilever is proportional to the gradient of force acting on the tip $[9,11,12]$. In this study, measurements were done using scanning probe microscope NanoWizard (JPK Instruments, UK) with a pyramidal-tipped cantilever PPPMFMR-10 (Nanosensors, Switzerland) for MFM study and a high resolution tip DP14/Hi'Res-W/AIBS (Mikromasch, Bulgaria) for AFM measurements. MFM measurements were done in large applied field of $0.58 \mathrm{~T}$ able to magnetize nanoparticles close to their saturation level and keep magnetization in the direction perpendicular to the surface of the slides.

\section{THEORETICAL TREATMENT}

\section{A. Treatment of grey-scale images of the phase shift}

The resulting phase shift images from the experiments with the magnetic and non-magnetic tip were saved as $512 \times$ 512 pixels gray-scale images and processed with the software MATLAB R2020a. The quantitative analysis of the MFM measurements of the samples of Pd-Fe loaded bacteria was done by plotting experimental phase shift values over the surface of the Pd-Fe NPs as function of the tip lift height. The shift in phase is a result of attractive and repulsive forces acting on the tip, which are dependent on its perpendicular (z) distance from the surface.

The grey-scale images were loaded and cropped defining the same area in different slices to facilitate pixels selection. One pixel on the surface of four specific Pd-Fe NPs in the sample was selected on each z-image for all the measured distances. The intensity values of the selected pixels were extracted and transformed from the brightness into relative phase shift. This is an inverse procedure to that used by instrument converting the phase shift into brightness using unified multiplier and shift to produce grey-scale images.

\section{B. Simulation of the interaction of tip with magnetic nano- sphere}

The simulation of the interaction of the tip with a nanoparticle was performed by finite element analysis, with COMSOL Multiphysics (COMSOL, Inc.) software, assuming that a nanoparticle is spherical with diameter of 15 $\mathrm{nm}$. Inside the spherical particle, there exists a homogenous magnetic field $\mathrm{B}$, as well as homogeneous auxiliary magnetic field $\mathrm{H}$ and magnetization $\mathrm{M}$. The auxiliary field $\mathrm{H}$ has two components: the externally applied field $\mathrm{H}_{0}$, that determines $M$ according to the magnetization curve, and the demagnetizing field, which, for a spherical particle, cancels $1 / 3$ of $\mathrm{M}$. After calculating the resulting magnetic field $\mathrm{B}=$ $\mu_{0}(\mathrm{H}+\mathrm{M})$ inside the particle, it is straightforward to reconstruct the magnetic field B outside it. Simulations were done setting $\mathrm{B}$ inside the particle to the values of $0.1,0.2$, $0.3,0.4$ and 0.5 T. Fig. 1 shows an example of the distribution of $\mathrm{B}$ around the nanoparticle at both $\mu_{0} \mathrm{H}_{0}$ and $\mathrm{B}$ inside it of $0.1 \mathrm{~T}$. For the force acting on the tip, only the gradient of $\mathrm{B}$ component along the direction of applied field is important, assuming a fixed magnetization of the tip in the same direction. The homogeneous part of B is not important, as it does not impose force on the tip.

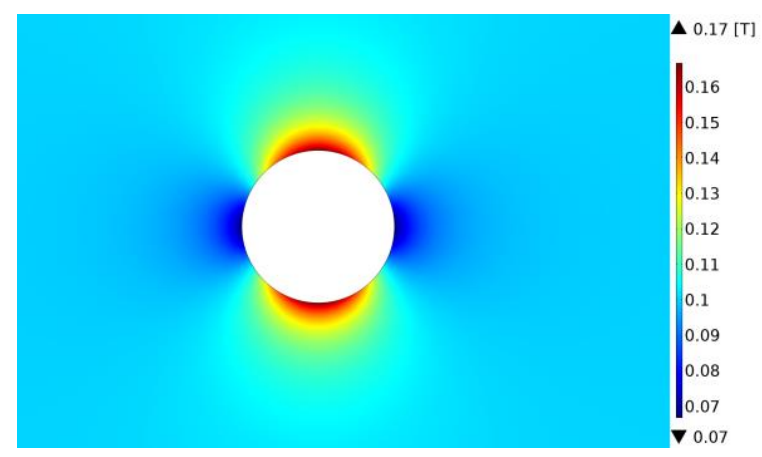

Fig. 1. Distribution of $\mathrm{B}$ around the nanoparticle in a cross-section perpendicular to the surface of the sample at both $\mu_{0} \mathrm{H}_{0}$ and $\mathrm{B}$ inside it of 0.1 $\mathrm{T}$. 


\section{RESULTS AND DISCUSSION}

\section{A. Comparison of measurements with magnetic and non- magnetic tip}

Fig. 2 shows some representative grey-scale images of the phase shift measured in a $5 \mu \mathrm{m} \times 5 \mu \mathrm{m}$ area, recorded with the non-magnetic tip (Figs. 2a, 2b) at heights of 5 and $12 \mathrm{~nm}$, and with a magnetic tip (Figs. 2c, 2d) at heights of 22 and $50 \mathrm{~nm}$, respectively. The area contains two crosssections of bacteria situated closer to the bottom of the images, and a large cluster of nanoparticles attached to the top of the bacterium in the bottom-right corner. The images show gradient of force acting to the tip. Dark and bright shades represent attraction and repulsion, respectively.

The main difference found between scans with nonmagnetic (Figs. 2a, 2b) and magnetic (Figs. 2c, 2d) tips was that in the former case, the measurable magnetic signal lasted up to a lift height of $22 \mathrm{~nm}$ above the surface, while in the latter, up to $400 \mathrm{~nm}$. This is in agreement with the expected behavior of short-range van der Waals force and long-range magnetic forces, because the latter can only be measured with the magnetic tip. At large heights, the signal becomes diffused (Fig. 2d), while at low heights individual nanoparticles can be resolved quite well (Figs. 2a, 2c). The results also present two kinds of nanoparticles. For most of them, at low heights attraction of the tip is changed to repulsion (see bright dots inside black spots in Fig. 2a). Presumably, these nanoparticles are bulging above the surface. There are however, a few nanoparticles, which do not demonstrate repulsion even at lowest heights.
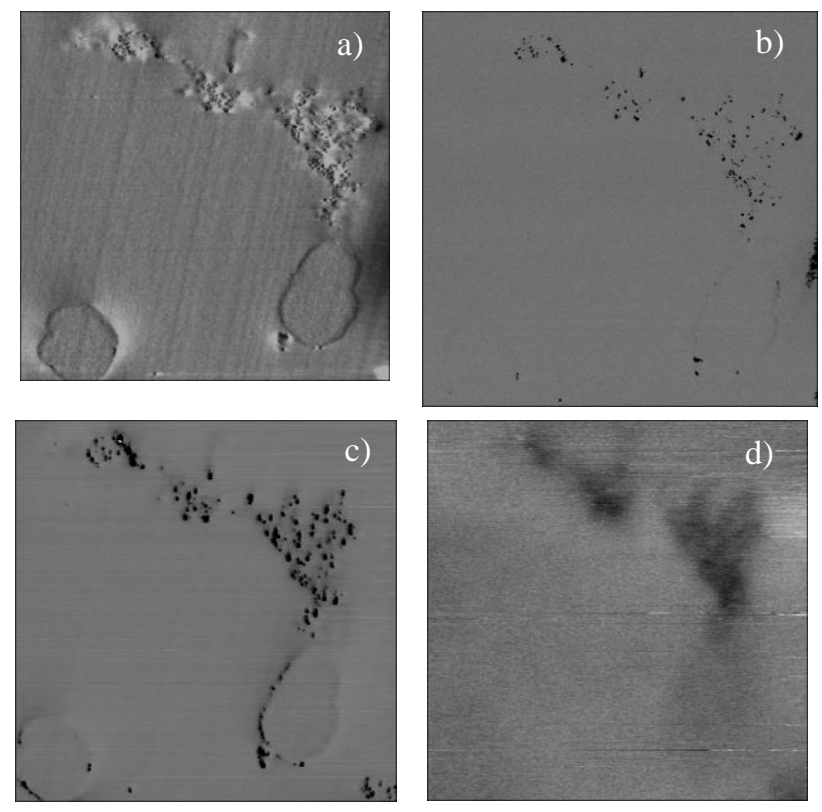

Fig. 2. Representative grey-scale phase-shift images of the area $5 \mu \mathrm{m} \times 5 \mu \mathrm{m}$ recorded with non-magnetic tip (a, b) at the heights of 5 and $12 \mathrm{~nm}$, respectively, and with magnetic tip (c, d) at heights of 22 and $50 \mathrm{~nm}$, respectively.

Fig. 3 shows the results obtained by the computer treatment of the light intensity in the grey-scale images for the four randomly selected nanoparticles. For these nanoparticles, it is possible to see a switching behavior from attraction to repulsion as the tip gets closer to the surface. In the plot, the force gradient sign is opposite to the phase shift (negative value of phase shift corresponds to attraction). In such a representation, obtained curves resemble the standard van der Waals curve. Measurements with magnetic tip are shown by black, wine, green and magenta squares, while the measurements with non-magnetic tip for the same nanoparticles are all shown in blue color.

In spite of significant scattering of the points that belong to different nanoparticles, it is clear from the plot that van der Waals force, at the height of about $22 \mathrm{~nm}$, are reduced to zero. Only the noise was registered above this height. Instead, attractive magnetic forces last until much bigger heights. In the case of the magnetic tip, the plotted transition from attraction to repulsion takes place at the height of about $22 \mathrm{~nm}$, at which van der Waals force is added to magnetic forces.

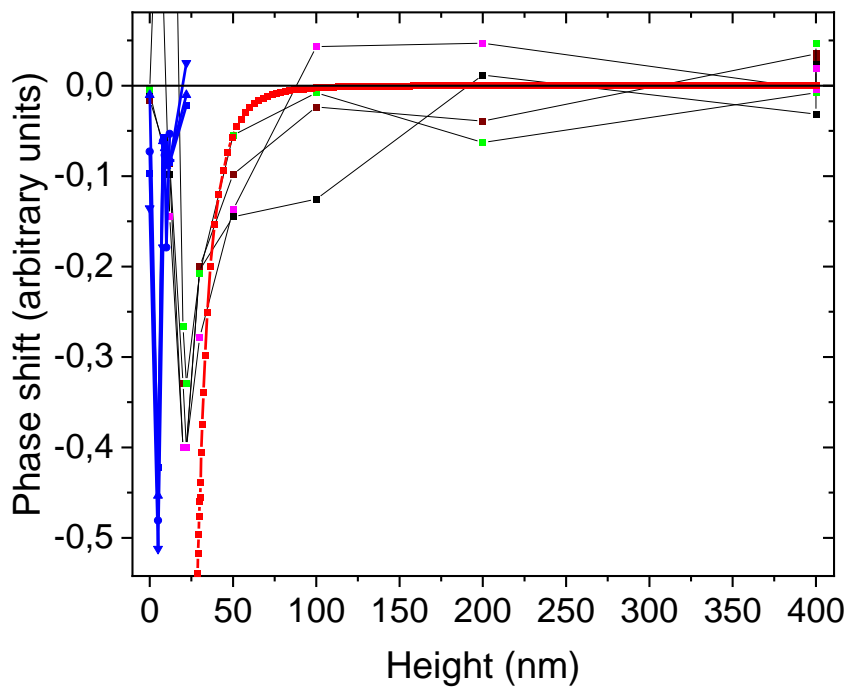

Fig. 3. Treated intensity of the light in the grey-scale images for four selected nanoparticles showing phase shift as function of height above the sample, recorded with magnetic (black, wine, green and magenta squares) and non-magnetic (blue symbols) tip. The line with red squares shows the simulated curve for the interaction of a spherical particle, of the diameter of $15 \mathrm{~nm}$, with the magnetic tip.

\section{B. Comparison with simulation}

The red curve in Fig. 3 presents the simulated interaction of the magnetic tip with a spherical particle of the diameter of $15 \mathrm{~nm}$. It is a generic curve, to which all simulated results collapse after being scaled with the magnetic field inside the particle. Although the tendency of the decay in the gradient of magnetic force (or phase shift) with height is correct, the large scattering of the data does not allow accurate comparison of the experiment with simulation.

However, this correlation was easier to establish with the second type of nanoparticles that do not show transition from attraction to repulsion. Most likely, these nanoparticles are slightly below the surface of the sample. Fig. 4 shows the phase shift as function of the lift height for one of these nanoparticles recorded with magnetic tip. The agreement with simulation here is satisfactory. The deviation at low heights is likely to be due to the growing contribution of van der Waals force. 


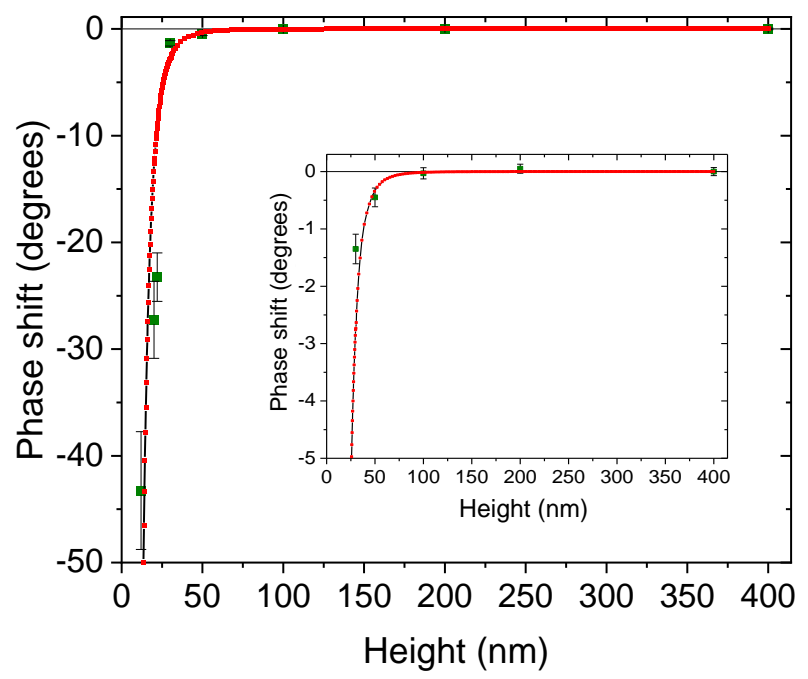

Fig. 4. Phase shift as function of height for a nanoparticle not showing transition from attraction to repulsion at low heights, recorded with a magnetic tip. The line with red squares shows the simulated curve for the interaction of a spherical particle of the diameter of $15 \mathrm{~nm}$ with the magnetic tip. Inset show better resolved data of the same plot at larger heights.

\section{Magnetic mapping}

From Fig. 3, it is clear that in order to record a pure or dominant magnetic signal, one needs the use of a two-pass technique at a height above $22 \mathrm{~nm}$, for this sample, or the height, where the gradient of van der Waals force is negligible. However, using a large lift value is also not desirable, since magnetic signals from different nanoparticles merge and the image becomes diffused. The signals also become weaker and difficult to separate from the background noise. Therefore, the optimal height should be as close as possible to the lift height of the van der Waals force recession.

Using this rule, several magnetic maps have been recorded. Fig. 5 shows one of such maps with visible crosssections of several bacteria and magnetic nanoparticles inbetween. Some nanoparticles look spherical and many of them display effect of the transition from attraction to repulsion as evidenced by the bright spots inside.

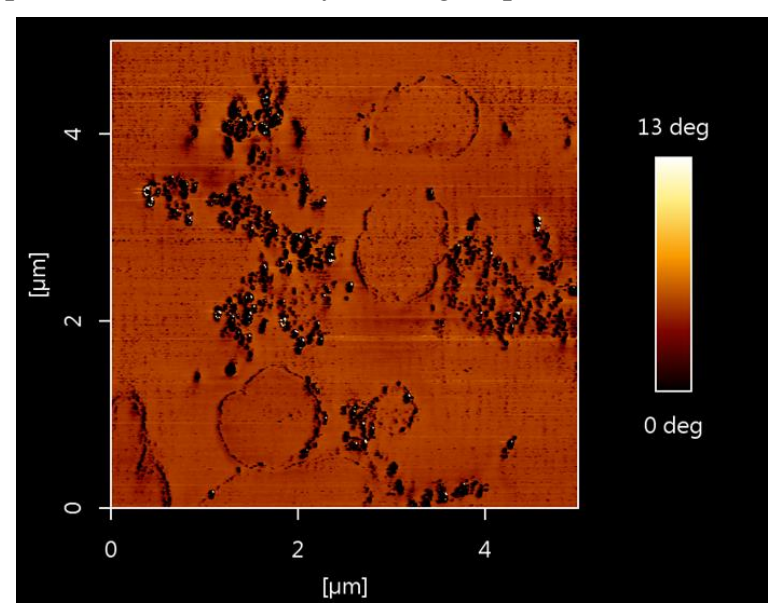

Fig. 5. Magnetic map at lift heigth $22 \mathrm{~nm}$ of the cross-sections of several bacteria with reduced from the solution $\mathrm{Pd}-\mathrm{Fe}$ magnetic particles inbetween.
The area of the map in Fig. 5 is $5 \mu \mathrm{m} \times 5 \mu \mathrm{m}$, just like in Fig. 2. There is the possibility, however, to visualize magnetism in much smaller areas. An example is the magnetic map of an individual nanoparticle shown in Fig. 6. This nanoparticle is not spherical, which we presume, could be another source of deviation of the experimental points from the simulated curve, similar to what takes place in Figs. 3 and 4.

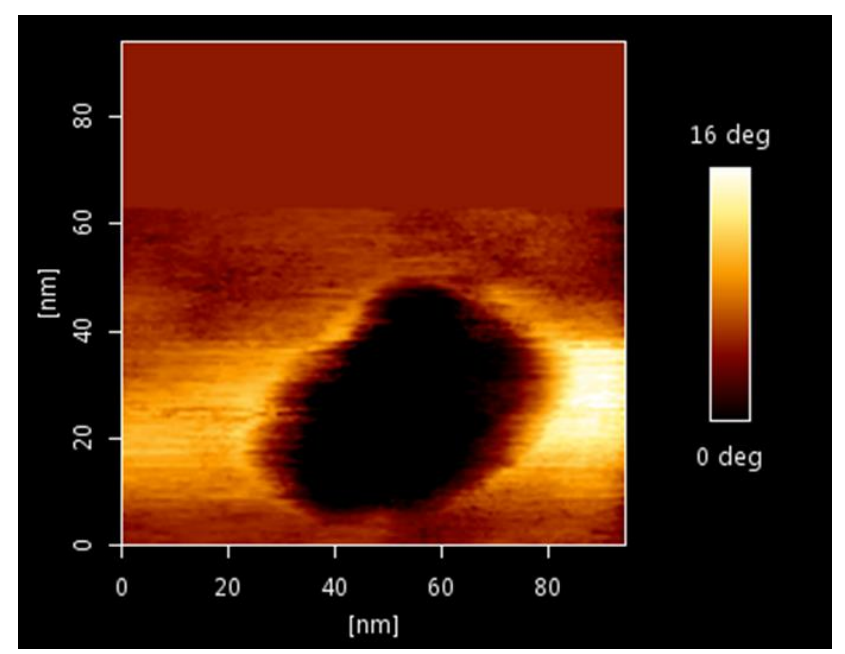

Fig. 6. High-resolution magnetic map of an individual Pd-Fe magnetic nanoparticle recorded at height of $22 \mathrm{~nm}$ above sample surface.

\section{CONCLUSIONS}

Currently, the complete characterization of the biological pathways of magnetic nanoparticles production remains to be fully understood. MFM could be employed as a technique to study their magnetic properties in samples, which simultaneously locates their position within or outside the microorganisms. In this study, for MFM measurements, the tip is lifted off the surface in order to separate the short-range forces from the long-range forces. This allowed the detailed study of the magnetism of nanoparticles produced by the bacteria.

The final data present relatively high scattering due to the difference in the behavior of nanoparticles and following the way the information was processed. Nevertheless, it was still possible to correlate data with the simulations results and observe the phase shift controlled by the transition from the attraction to the repulsion at the decrease of the height above the sample. The particles that do not show transition to the repulsion demonstrate good agreement with the simulations. The measurements of the same area with magnetic and non-magnetic tips allowed to find a condition at which pure or dominant magnetic response could be measured. Following this condition, magnetic maps of several bacterial cross sections with recovered $\mathrm{Pd}-\mathrm{Fe}$ nanoparticles have been recorded.

\section{ACKNOWLEDGMENT}

This work is part of the Project "BEDPAN - Bioengineered Palladium Nanoparticles", funded by the Research Council of Norway, RCN294605. 


\section{REFERENCES}

[1] T. Taniyama, E. Ohta, and T. Sato, "Observation of $4 \mathrm{~d}$ ferromagnetism in free-standing Pd fine particles," Europhysics Letters, vol. 38, pp. 195-200, April 1997.

[2] I. P. Mikheenko, P. M. Mikheenko, C. N. W. Darlington, C. M. Muirhead, and L. E. Macaskie, "Magnetic testing of Pd loaded bacteria," in Process Metallurgy 11 A, part B: Biosorption and Bioremediation, Elsevier Science, 2001, pp. 525-532.

[3] P. Bera and M. S. Hegde, "Recent advances in auto exhaust catalysis," Journal of the Indian Institute of Science, vol. 90, pp. 299-305, April-June 2010.

[4] M. A. Miller, B. Askevold, H. Mikula, R. H. Kohler, D. Pirovich, and R. Weissleder, "Nano-palladium is a cellular catalyst for in vivo chemistry," Nature Communications, vol. 8, pp. 1-13, July 2017.

[5] F. Ghandehari, M. Fani, and M. Rezaee, "Biosynthesis of Iron Oxide Nanoparticles by Cytoplasmic Extract of Bacteria Lactobacillus Fermentum," Journal of Medicinal and Chemical Sciences, vol. 1, pp. 28-30, October 2018.

[6] P. Pourali and B. Yahyaei, "Biological production of silver nanoparticles by soil isolated bacteria and preliminary study of their cytotoxicity and cutaneous wound healing efficiency in rat," Journal of Trace Elements in Medicine and Biology, vol. 34, pp. 22-31, March 2016.

[7] A. Weis, S. Colombo, V. Dolgovskiy, Z. D. Grujić, V. Lebedev, and J. Zhang, "Characterizing and imaging magnetic nanoparticles by optical magnetometry," in Journal of Physics: Conference Series, vol. 793, 012032, February 2017.

[8] G. J. Nieuwenhuys, "Magnetic behaviour of cobalt, iron and manganese dissolved in palladium," Advances in Physics, vol. 24, pp. 515-591, January 1975.

[9] D. Passeri, C. Dong, M. Reggente, L. Angeloni, M. Barteri, F. A. Scaramuzzo, F. De Angelis, F. Marinelli, F. Antonelli, F. Rinaldi, C. Marianecci, M. Carafa, A. Sorbo, D. Sordi, I. WCE Arends, and M. Rossi, "Magnetic force microscopy: Quantitative issues in biomaterials," BioMatter, vol. 4, e29507, pp. 1-16, July 2014,

[10] G. Cordova, B. Y. Lee, and Z. Leonenko, "Magnetic force microscopy for nanoparticle characterization," NanoWorld Journal, vol. 2, pp. 10-14, April 2017.

[11] B. Torre G. Bertoni, D. Fragouli, A. Falqui, M. Salerno, A. Diaspro, R. Cingolani, and A. Athanassiou, "Magnetic force microscopy and energy loss imaging of superparamagnetic iron oxide nanoparticles," Scientific Reports, vol. 1, pp. 1-8, December 2011.

[12] D. Sarid, Scanning Force Microscopy: With Applications to Electric, Magnetic, and Atomic Forces. New York: Oxford University Press, 1994. 